\title{
ADAPTABLE TEST-BED FOR CHARACTERIZATION OF MICRO-WINEGLASS RESONATORS
}

D. Senkal, M.J. Ahamed, A.A. Trusov, and A.M. Shkel

MicroSystems Laboratory, University of California, Irvine, CA, USA

\begin{abstract}
We present an adaptable test-bed for characterization of 3-D micro-wineglass resonators. The test-bed provides two interchangeable modes of excitation: (1) Mechanical pinging using a piezo actuated probe assembly, (2) electrostatic excitation using assembled electrode structures with $<20 \mu \mathrm{m}$ capacitive gaps. Two modes of detection is also available: Optical pick-up using laser Doppler vibrometry and capacitive detection. 3-D microglassblown wineglass resonators were used to demonstrate the capabilities of the test-bed. Electrostatic excitation and capacitive detection was used to obtain the frequency response of a glass resonator showing a Q-factor of 40,000 at $14.8 \mathrm{kHz}$. Piezo-pinging was used to obtain the time domain response of a resonator, showing a $28 \mathrm{~Hz}$ frequency split $(0.1 \%$ relative split) between the two degenerate wineglass modes $\left(f_{1}=22036 \mathrm{~Hz}, f_{2}=22064 \mathrm{~Hz}\right)$.
\end{abstract}

\section{INTRODUCTION}

There is a growing interest in 3-D MEMS wineglass resonators for the next generation timing, signal processing, and inertial sensing applications due to potential advantages of the wineglass geometry in terms of symmetry, minimization of energy loss, thermal stability, and immunity to external vibrations [1]. However, difficulties associated with fabricating smooth, symmetric, high aspect ratio 3-D structures have so far prevented their integration with MEMS techniques.

With the emergence of new 3-D micro-machining techniques, batch fabrication of 3-D MEMS wineglass structures is becoming feasible. For instance, hemispherical shells fabricated by deposition of polysilicon [2] or silicon nitride [3] thin films into isotropically etched cavities have recently been demonstrated. Alternative fabrication techniques include thin film deposition onto high-precision ball bearings [4], "3-D SOULE" (micro ultrasonic machining, lapping, and micro electro-discharge machining) process for fabrication of mushroom and concave shaped spherical structures [5], as well as the pressure / surface tension driven fabrication techniques such as blow molding of bulk metallic glasses into pre-etched cavities [6] and micro-glassblowing of borosilicate glass [7-11] or fused silica [12-13] into spherical or wineglass geometries.

Despite the recent advances in micro-wineglass resonator fabrication, the technology development is in its infancy and characterization of the early stage prototypes presents a challenge. This paper introduces

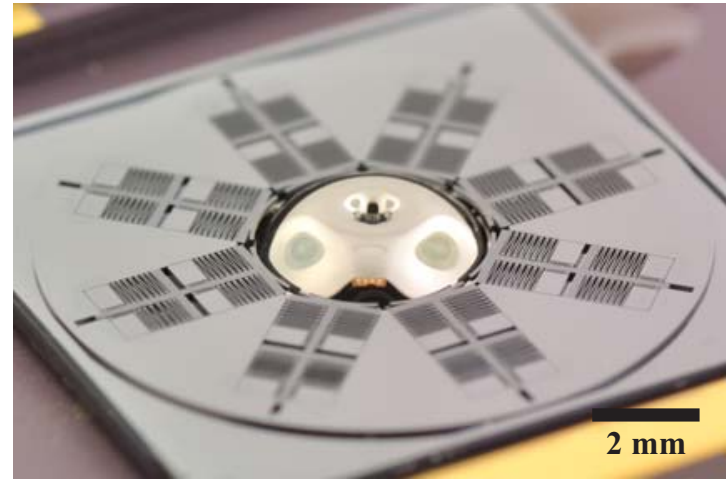

Figure 1: Electrode structures assembled onto a micro-glassblown wineglass resonator with $<20 \mu \mathrm{m}$

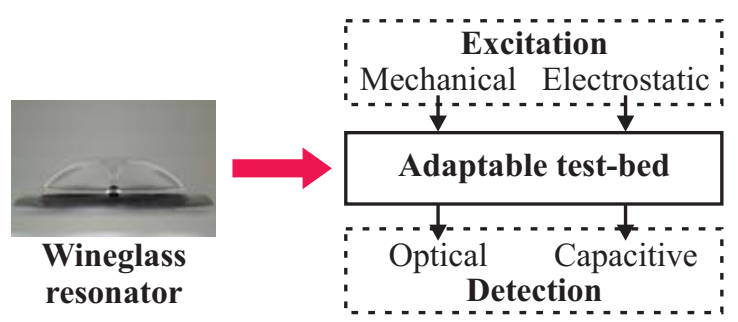

Figure 2: Schematic description of adaptable test-bed for characterization of micro-wineglass prototypes.

a test-bed with interchangeable modes of excitation and detection for characterization of micro-wineglass devices of any shape or size, eliminating the need for in-situ electrode fabrication during the development cycle of the resonator architecture, Figure 1. Additionally, mechanical excitation capability eliminates the need for conductive coating of dielectric resonators, electrical feed-throughs or DC biasing, all of which can obscure the actual resonator performance.

The test-bed provides two interchangeable modes of excitation: Mechanical pinging and electrostatic excitation, Figure 2. Mechanical pinging is provided by a piezo actuated probe assembly, whereas electrostatic excitation is provided by assembled electrode structures with $<20 \mu \mathrm{m}$ capacitive gaps. Two modes of detection is also available: Optical pick-up and capacitive detection, Figure 2. Optical pick-up is provided by laser Doppler vibrometry and capacitive detection is provided by assembled electrode structures. 3D micro-glassblown wineglass resonators were used to demonstrate the capabilities of the test-bed in extracting resonator parameters such as Q-factor or frequency split between degenerate wineglass modes. 


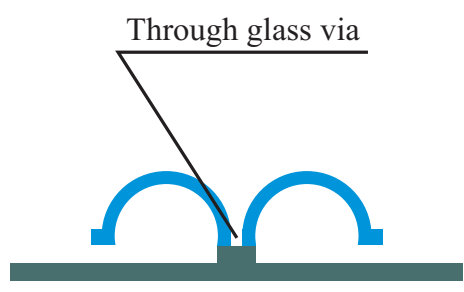

a) Glassblown wineglass

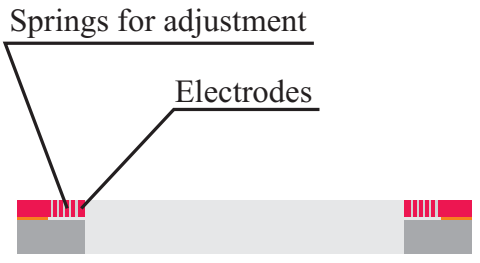

b) Adjustable electrodes
Electrodes adjusted to wineglass

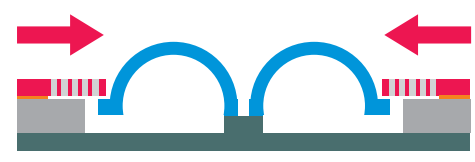

c) Wineglass \& assembled electrodes

Figure 3: Electrodes are fabricated on an SOI wafer, bonded onto the resonator wafer and adjusted.

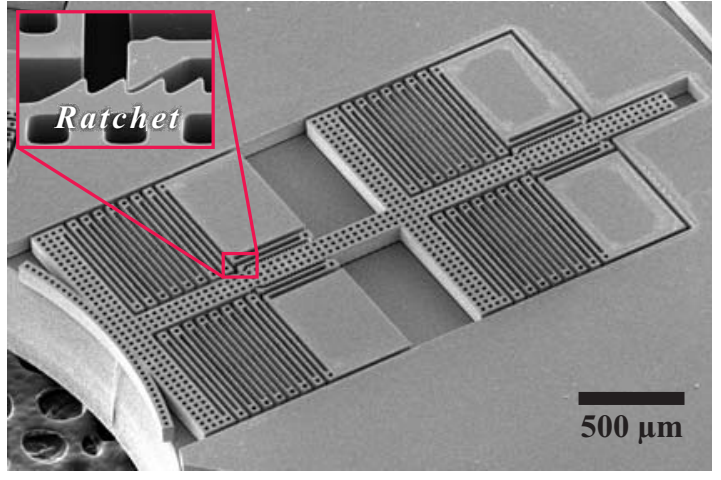

Figure 4: SEM image of an adjustable electrode with $400 \mu \mathrm{m}$ maximum displacement and $10 \mu \mathrm{m}$ positioning resolution.

\section{ASSEMBLED ELECTRODES}

Electrostatic excitation and detection is provided by assembled electrode structures, which are fabricated separately from the resonator on a SOI wafer, Figure 3. The SOI stack consists of $500 \mu \mathrm{m}$ silicon substrate layer, $5 \mu \mathrm{m}$ buried oxide layer and $100 \mu \mathrm{m}$ silicon device layer. Each electrode assembly consists of 8 independent electrodes that are spaced $45^{\circ}$ apart around a central thru-hole. Large spring structures on each electrode allow an adjustment distance up to $400 \mu \mathrm{m}$ for each individual electrode (total of $800 \mu \mathrm{m}$ along the resonator diameter), which permits a single electrode design to be used for different wineglass architectures and diameters, Figure 4. Electrode assemblies with 7 different central-hole diameters were fabricated on the same SOI wafer, which covers all wineglass diameters from $1 \mathrm{~mm}$ to $4.5 \mathrm{~mm}$.

The fabrication of SOI electrodes start by lithographically patterning the back-side thru-hole and etching using DRIE to a depth of $500 \mu \mathrm{m}$. This is followed by patterning and etching the device layer to create the electrode structures. For the electrode structures, the DRIE is performed to a depth of $100 \mu \mathrm{m}$, using a $1.6 \mu \mathrm{m}$ oxide hard-mask for better feature resolution, then $\mathrm{O}_{2}$ ashing / RCA-1 cleaning is used to get rid of excess photoresist and etch residues. The final step of the fabrication process is HF wet etching of buried oxide layer to release the electrode structures.

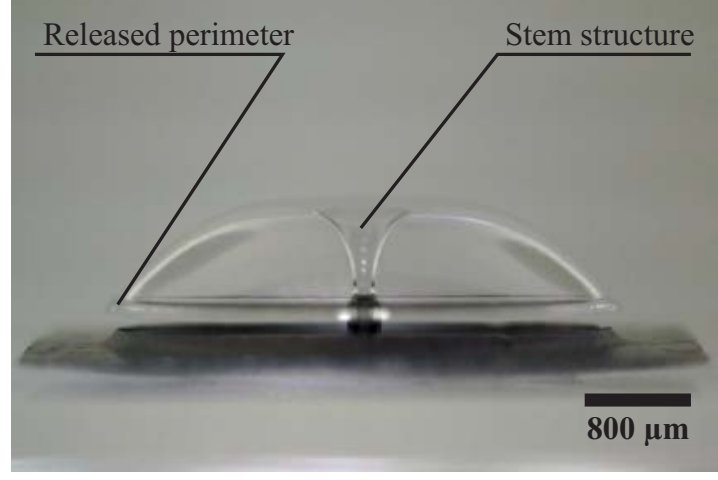

Figure 5: Released wineglass structure with $4.2 \mathrm{~mm}$ diameter, $50 \mu \mathrm{m}$ thickness and $300 \mu \mathrm{m}$ central stem.

\section{OPERATION}

Once the fabrication is complete the electrode structures are singulated and bonded onto the microwineglass die, Figure 3. Then, each electrode is pushed to close proximity of the wineglass by using a micromanipulator. When the correct location is achieved four ratchet mechanisms ( 2 front, 2 back) keep the electrodes in place. The ratchet mechanisms act on 2 rack gears placed on the electrode. The pitch distance on the rack gears are $20 \mu \mathrm{m}$. By offsetting the front and rear ratchet mechanisms by $10 \mu \mathrm{m}$ relative to the teeth pitch of the rack gear, a positioning resolution of $10 \mu \mathrm{m}$ was obtained (minimum capacitive gap).

For experimental characterization, assembled electrodes were bonded onto micro-glassblown wineglass structures, Figure 5. A wineglass with $4.2 \mathrm{~mm}$ diameter, $50 \mu \mathrm{m}$ thickness, and $300 \mu \mathrm{m}$ central stem was tested using an assembled electrode structure with $4.5 \mathrm{~mm}$ thru-hole. The entire assembly was placed into a ceramic DIP package with gold backplate and wirebonded at the anchors of each electrode. The bias voltage to the resonator is applied through the gold back-plate, which connects to the resonator die, through it to the via at the center of the stem and to the resonator metal layer.

Frequency sweeps were obtained using an Agilent 4395A network analyzer. Two opposite electrodes were used with the goal of forced excitation of the $n=2$ wineglass mode. A DC voltage of $10 \mathrm{~V}$ and $\mathrm{AC}$ voltage of $5 \mathrm{~V}_{\mathrm{pp}}$ were used. A Q-factor of 40,000 was observed at $14.833 \mathrm{kHz}$, giving a time decay constant of $\sim 0.9$ seconds, Figure 6 . 


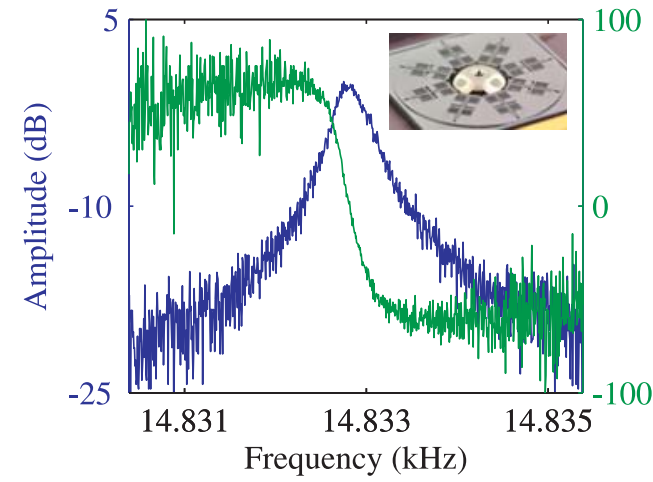

Figure 6: Electrostatic frequency sweep using assembled electrode structures, showing $Q=40 \mathrm{k}$ at $14.8 \mathrm{kHz}$.

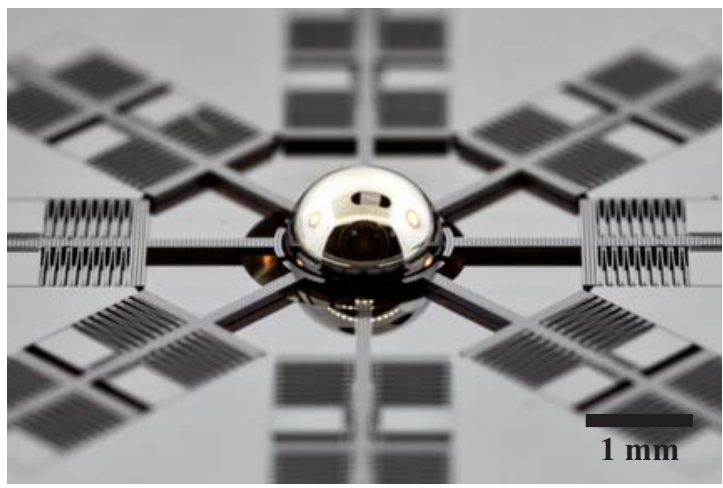

Figure 7: A glassblown spherical resonator with assembled electrodes. Diameter is $1.2 \mathrm{~mm}$ and thickness is $\sim 5 \mu \mathrm{m}$.

Adjustable nature of the assembled electrode structures allow, characterization of 3-D resonator structures with varying size and diameter. Figure 7 shows assembled electrodes structures around a $1.2 \mathrm{~mm}$ micro-glassblown spherical resonator with resonant frequencies in the $\mathrm{MHz}$ range.

\section{PIEZO PINGER SETUP}

Capacitive transduction requires an electrical connection to the resonator body, which can be achieved either by using a conductive resonator material or coating the resonator with a conductive layer. If the resonator is made out of a dielectric material, such as $\mathrm{SiO}_{2}$, conductive coating with an appropriate feed-through might not be available during early stages of the resonator fabrication. Otherwise, it might be undesirable to use capacitive transduction for testing, since factors such as electrostatic coupling effect or metallization losses can obscure the actual mechanical characteristics of the wineglass resonator. For these reasons a mechanical excitation method that can operate under vacuum and directionally excite the wineglass modes is highly desirable as an alternative to electrostatic transduction.

A piezo-pinger setup was constructed using offthe-shelf components and rapid prototyped fixtures.

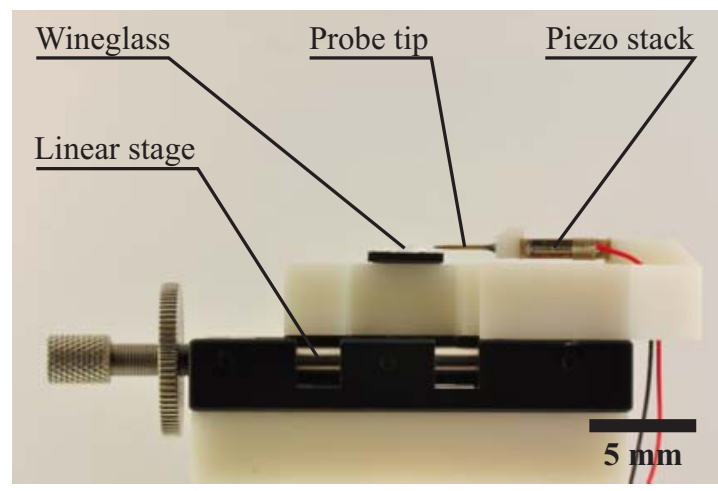

Figure 8: Piezo-pinger setup for time domain characterization, piezo-stack is actuated to probe the wineglass resonator.

The piezo-pinger assembly consists of 3 main components: A linear micro-stage with a positioning accuracy of $1 \mu \mathrm{m}$, a high displacement $(32 \mu \mathrm{m})$ piezostack, and a tungsten probe tip (100 $\mu \mathrm{m}$ tip radius), Figure 8 . The setup is built with vacuum compatible components and fits into a bounding box of $80 \mathrm{~mm} \mathrm{x}$ $30 \mathrm{~mm} \times 20 \mathrm{~mm}$.

\section{OPERATION}

Wineglass resonator is placed on the linear stage and kept in place using a non-permenant bonding method, such as indium or double sided kapton tape. Linear stage is adjusted under a microscope until the probe tip is within 5-10 $\mu \mathrm{m}$ proximity of the wineglass. Then, the piezo-pinger is placed into the vacuum chamber and connected to a high-voltage power supply. A mechanical switch with $1 \mathrm{k} \Omega$ discharge resistor was used for controlling the piezostack. A Polytec Laser Doppler Vibrometer pointed onto the outer edge of the wineglass resonator through the optical port of the vacuum chamber is used for detection.

Experiment starts by increasing the voltage on the piezo-stack until the probe tip barely makes contact with the edge of the wineglass (up to $100 \mathrm{~V} \mathrm{DC}$ ), which applies a point load onto the wineglass resonator. At $\mathrm{t}=0$ the probe tip is retracted by shorting the piezo-stack over the $1 \mathrm{k} \Omega$ discharge resistor, which leaves the wineglass free to vibrate. Time domain response is captured using the laser Doppler vibrometer at a sampling rate of $1256 \mathrm{kHz}$. The data can later be analyzed either in the time domain or in frequency domain using a Fast Fourier Transform (FFT) algorithm.

Time domain response, in Figure 9, was obtained on a wineglass resonator using the described piezopinger setup and laser Doppler vibrometer detection. Figure 9 shows a $28 \mathrm{~Hz}$ beat signal created by the frequency split between two degenerate wineglass modes. Post-processing using a FFT algorithm confirms the frequency split of $28 \mathrm{~Hz}$ at resonant frequencies $f_{1}=22036 \mathrm{~Hz}$ and $f_{2}=22064 \mathrm{~Hz}$. 


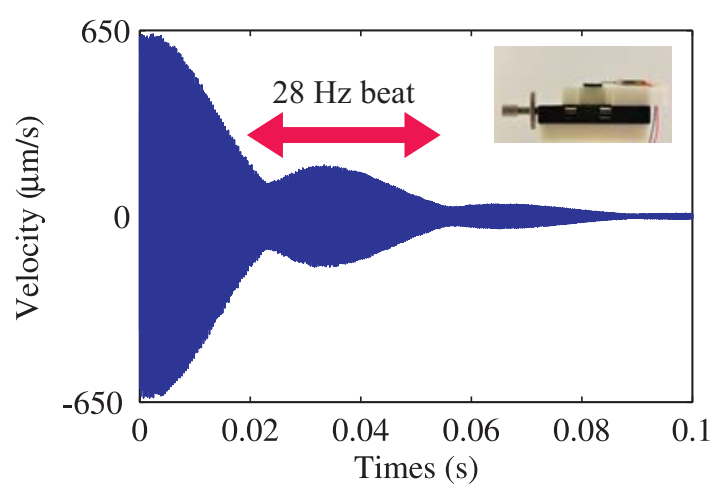

Figure 9: Time decay response obtained by retracting the probe tip, beat signal between two degenerate modes shows $\Delta f=28 \mathrm{~Hz}$.

\section{CONCLUSIONS}

Electrostatic excitation has advantages in terms of mode selectivity as unwanted modes can be suppressed by using a combination of balanced electrodes. However, it can potentially obscure the actual resonator behavior due to metallization losses or the electrostatic coupling effect. Both of these effects can be avoided by using a purely mechanical excitation method, such as piezo-pinging. Another benefit of the piezo-pinger setup is the non-permanent attachment of the resonator, which allows quick testing of multiple wineglass resonators. A comparative summary of the two excitation methods is presented in Table 1.

Table 1: Comparison of excitation methods.

\begin{tabular}{lcc}
\hline & $\begin{array}{c}\text { Assembled } \\
\text { electrodes }\end{array}$ & Piezo-pinger \\
\hline Attachment & Permanent & Non-permanent \\
Coating & Metalized & None required \\
Detection & Electrostatic & Laser vibrometer \\
Mode selection & Yes & No \\
\hline
\end{tabular}

By decoupling the resonator development from electrode fabrication, the test-bed allows greater flexibility in design and fabrication. In addition, the testbed presented in this paper is universally applicable to any micro-wineglass resonator, regardless of shape, size or fabrication process involved.

\section{ACKNOWLEDGEMENTS}

This material is based upon work supported by DARPA/SPAWAR under Grant N66001-10-1-4074 (Program Manager Dr. Tayo Akinwande) and DARPA grant W31P4Q-11-1-0006. Devices were designed and tested in UCI MicroSystems Lab. Authors would like to thank UCI INRF staff Jake Hes, Mo Kebaili, $\mathrm{Vu}$ Phan and Lifeng Zheng for their help and valuable suggestions on the fabrication aspects of the project.

\section{REFERENCES}

[1] D.M. Rozelle, "The hemispherical resonator gyro: From wineglass to the planets", in Proc. AAS/AIAA Space Flight Mechanics Meeting, Feb. 2009, pp. 1157-1178.
[2] L.D. Sorenson, X. Gao, F. Ayazi, "3-D Micromachined Hemispherical Shell Resonators With Integrated Capacitive Transducers," Proc. IEEE MEMS'12, Paris, France, Jan. 29 - Feb. 2, 2012, pp. 168-171.

[3] C.L. Fegely, D.N. Hutchison, S.A. Bhave, "Isotropic etching of 111 SCS for wafer-scale manufacturing of perfectly hemispherical silicon molds," Proc. Transducers'11, Beijing, China, June 59, 2011, pp 2595-2598.

[4] P. Pai, F. Chowdhury, C. Mastrangelo, M. TabibAzar, "MEMS-based Hemispherical Resonator Gyroscopes", Proc. IEEE Sensors 2012, Taipei, Taiwan, October 29-31, 2012.

[5] K. Visvanathan, Li Tao, Y. B. Gianchandani, "3D-soule: A fabrication process for large scale integration and micro-machining of spherical structures", Proc. IEEE MEMS'11, Jan. 21 - 27, 2011, pp. 45-48.

[6] B. Sarac, G. Kumar, T. Hodges, S. Ding, A. Desai, J. Schroers, "Three-Dimensional Shell Fabrication Using Blow Molding of Bulk Metallic Glass," IEEE J. Microelectromech. Syst., vol. 20, no. 1, pp. 28-36, 2011.

[7] E. J. Eklund and A. M. Shkel, "Method and Apparatus for Wafer-level Micro-glass-blowing," US Patent 7694531, Issued April 13, 2010.

[8] E.J. Eklund, A.M. Shkel, "Glass Blowing on a Wafer Level," IEEE J. Microelectromech. Syst., 16(2), pp. 232-239, 2007.

[9] I.P Prikhodko, S.A. Zotov, A.A. Trusov A.M. Shkel, "Microscale Glass-Blown Three-Dimensional Spherical Shell Resonators," IEEE J. Microelectromech. Syst., vol. 20, no. 3, pp.691-701, 2011.

[10] S. A. Zotov, I. P. Prikhodko, A. A. Trusov, and A. M. Shkel, "3-D Micromachined Spherical Shell Resonators with Integrated Electromagnetic and Electrostatic Transducers," in Solid-State Sensors, Actuators, and Microsystems Workshop 2010, 2010, pp. 11-14.

[11] D. Senkal, I. P. Prikhodko, A. A. Trusov, A. M. Shkel, "Micromachined 3-D Glass-Blown Wineglass Structures for Vibratory MEMS Applications," Technologies or Future Micro-Nano Manufacturing, Napa, California, USA, August 8 - 10, 2011, pp. 166169.

[12] D. Senkal, C. Raum, A. Trusov, and A. M. Shkel, "Titania Silicate/Fused quartz Glassblowing for 3-D Fabrication of Low Internal Loss Wineglass Micro-structures," in Solid-State Sensors, Actuators, and Microsystems Workshop 2012, 2012, pp. 267270.

[13] D. Senkal, M. J. Ahamed, A. Trusov, and A. M. Shkel, "High Temperature Micro-Glassblowing Process Demonstrated on Fused Quartz and ULE TSG," in Sensors and Actuators A: Physical, (In print).

\section{CONTACT}

A.A. Trusov, tel: +1-949-824-6314; atrusov@uci.edu. 